\title{
Long non-coding RNAs and genes contributing to the generation of cancer stem cells in hepatocellular carcinoma identified by RNA sequencing analysis
}

\author{
LIYE CAO $^{1 *}$, QUAN ZHANG ${ }^{2 *}$, SHUJIE CHENG $^{2}$, ZHI CHEN $^{2}$, \\ ZHIJUAN HUA ${ }^{2}$, JIHONG YANG ${ }^{2}, \mathrm{DAN} \mathrm{LIU}^{4}$ and $\mathrm{NACUI}^{3}$ \\ Departments of ${ }^{1}$ Medical Ultrasound, ${ }^{2}$ Hepatobiliary Surgery and ${ }^{3}$ Intensive Care Unit, \\ Affiliated Hospital of Hebei University, Baoding, Hebei 071000; ${ }^{4}$ Department of Ultrasonic Imaging, \\ Zhuhai People's Hospital, Zhuhai, Quangdong 519000, P.R. China
}

Received March 7, 2016; Accepted April 22, 2016

DOI: 10.3892/or.2016.5120

\begin{abstract}
Cancer stem cells (CSCs) play important roles in cancer initiation, progression and metastasis. The aim of the present study was to identify the potential targets that may contribute to the generation of hepatocellular carcinoma stem cells (HCSCs) from hepatocellular carcinoma (HCC) cells The RNA sequencing (RNA-Seq) dataset GSE70537 was downloaded from the Gene Expression Omnibus (GEO) database. Raw RNA sequences were mapped to the GRCh37/hg19 genome based on TopHat and assembled through Cufflinks. Cuffdiff of Cufflinks was used for the screening of differentially expressed genes (DEGs) in the two types of HCSCs (Hep3B-C and Huh7-C) compared with the two types of HCC cells (Hep3B and Huh7) which were satisfied by the criteria of $\left|\log 2\left(\mathrm{RPKM}_{\mathrm{HCSC}} / \mathrm{RPKM}_{\mathrm{HCC}}\right)\right|>1$ and $\mathrm{p}<0.05$. In addition, based on the Database for Annotation, Visualization, and Integrated Discovery (DAVID), we screened the Gene Ontology (GO) terms and Kyoto Encyclopedia of Genes and Genomes (KEGG) pathways which were enriched in the DEGs. For the DEGs with consistent differential expression in the two lists of DEGs, the LncRNA2Target database was used for the identification of long non-coding RNA (lncRNA)-gene pairs. A total of 218 and 591 DEGs were identified for the Hep3B-C and Huh7-C samples, respectively, and 22 overlaps were obtained. Biological processes and pathways related to steroid biosynthesis/metabolism or other substance transport
\end{abstract}

Correspondence to: Dr Quan Zhang, Department of Hepatobiliary Surgery, Affiliated Hospital of Hebei University, 212 Yuhua East Road, Baoding, Hebei 071000, P.R. China

E-mail: zzttmm0312@163.com

*Contributed equally

Key words: cancer stem cells, RNA sequencing, Gene Expression Omnibus database, lncRNA were found to be enriched in the two lists of DEGs. Among the 22 overlaps, 16 were found to be consistently differentially expressed in the two HCSC samples, and the lncRNA-gene regulatory network of these genes was constructed. Moreover, several potential biomarkers that may play important roles in the transformation of HCSCs were identified in the regulation network. Through the bioinformatics analysis of the RNA-Seq dataset, several novel targets that were associated with the progression of HCC were obtained, and these targets may be valuable for the treatment and prognosis of HCC.

\section{Introduction}

Hepatocellular carcinoma (HCC) is one of the most common malignancies with a high patient mortality rate $(1,2)$. According to incomplete statistics, there are 360,000 diagnosed cases of primary liver cancer resulting in 350,000 deaths each year in China (3). Various new treatment options have been proposed and surgical resection is the most common strategy. Yet, the prognosis of HCC remains poor due to the difficulty in early detection and drug resistance (4-6). Thus, identification of biomarkers for the diagnosis and treatment of $\mathrm{HCC}$ is crucial.

Cancer stem cells (CSCs) are a type of cells associated with tumor initiation, metastasis and drug resistance (7-9). They are characterized by self-renewal capacity and the ability fo differentiate into multiple cell types. Moreover, they have been identified as therapeutic targets of many types of cancers, such as HCC (10). In a study by Sun et al, liver cancer stem-like cells (LCSCs) from MHCC97H cells were enriched and their aggressive phenotype compared with MHCC97H cells was confirmed. They also demonstrated their role in increased metastatic potential (11). By immunohistochemical methods, Matthai et al found that the LCSC marker, EpCAM, was downregulated in $\mathrm{HCC}$, which was not associated with hepatitis $\mathrm{B}$ virus (HBV) infection (12). Moreover, various genetic factors, such as gene mutation and exogenous stimulation, were also found to affect the properties of CSCs and thus cause the progression of cancers. Thus, investigation of the regulatory mechanisms of CSCs is necessary in order to improve the early detection, as well as the efficacious treatment of cancers. 
With the development of high-throughput technologies, such as microarray and RNA sequencing (RNA-Seq), more and more regulators of CSCs have been identified. Through small RNA-Seq, Jones et al found that CDX1-miR-215 regulated the differentiation of colorectal CSCs (13). Wang et al demonstrated the capability of $\mathrm{N}$-cadherin in the translation of CSCs in prostate cancer through ErbB signaling by microarray and western blot analysis (14). Long non-coding RNAs (lncRNAs) are non-protein coding transcripts which regulate gene expression in normal or cancer cells. In the past few years, many lncRNAs have been studied in LCSCs. They were shown to play important roles in the initiation, progression and metastatic of HCC. For example, lncRNA CUDR was found to regulate the malignant differentiation of LCSCs under the regulation of HULC and $\beta$-catenin (15). LncRNA HOTAIR was found to promote the growth of LCSCs via the downregulation of SETD2 (16). However, the exact mechanisms of CSCs in liver cancer cells remain unknown.

In the present study, using the RNA-Seq dataset in the Gene Expression Omnibus (GEO), we identified the differentially expressed genes (DEGs) in LCSCs compared with HCC cells. Function and pathway enrichment analysis indicated the Gene Ontology (GO) terms and pathways which may be involved in the process of translation from HCC cells into LCSCs. Moreover, an IncRNA-gene regulatory network screened out several regulatory relationships in LCSCs, which may contribute to the further understanding of the regulatory mechanisms of HCC.

\section{Materials and methods}

RNA-Seq dataset. The RNA-Seq dataset, GSE70537, which was deposited by Ding et al (8) was downloaded from GEO (http://www.ncbi.nlm.nih.gov/geo/). It contains two HCC samples (Hep3B, Huh7) and two hepatic cancer stem cell (HCSC) samples (Hep3B-C, Huh7-C). In this dataset, the total RNAs from the four samples were extracted and sequenced via Illumina Genome Analyzer II.

Differential expression analysis. TopHat (17) was used to map the RNA-Seq reads to the GRCh37/hg19 genome with the max mismatches of two. The sorted bam files from TopHat were used to calculate the numbers of the reads which were mapped to the exons of a gene based on Cufflinks (17) and normalized to reads per kilo-base of the exon model per million mapped reads (RPKM), which is the representation of the expression values of the genes. Finally, through Cuffdiff in Cufflinks, DEGs in HCSCs compared with HCC cells were obtained with the cutoff of $\left|\log 2\left(\mathrm{RPKM}_{\mathrm{HCSC}} / \mathrm{RPKM}_{\mathrm{HCC}}\right)\right|>1$ and $\mathrm{p}<0.05$.

Functional enrichment analysis. For the DEGs in HCSCs, the Database for Annotation, Visualization and Integrated Discovery (DAVID, https://david.ncifcrf.gov/) (18) was used for the functional and pathway enrichment analysis. In this study, GO terms and Kyoto Encyclopedia of Genes and Genomes (KEGG) pathways meeting $\mathrm{p}<0.05$ were considered to be enriched.

Screening of lncRNA-gene pairs. In the past few years, IncRNAs have been identified as important regulators in many
Table I. Total/mapped reads and mapping rates in every sample.

\begin{tabular}{lrrc}
\hline Sample ID & \multicolumn{1}{c}{$\begin{array}{c}\text { Total } \\
\text { reads }\end{array}$} & \multicolumn{1}{c}{$\begin{array}{c}\text { Mapped } \\
\text { reads }\end{array}$} & $\begin{array}{c}\text { Mapping } \\
\text { rates (\%) }\end{array}$ \\
\hline Hep3B & $9,347,804$ & $8,693,154$ & 93.0 \\
Huh7 & $11,367,875$ & $10,981,181$ & 96.6 \\
Hep3B-C & $7,842,223$ & $7,524,237$ & 95.9 \\
Huh7-C & $13,785,623$ & $13,276,070$ & 96.3 \\
\hline
\end{tabular}

phenotypes by knocking down or overexpressing them followed by microarray or RNA-Seq experiments. LncRNA2Target (http://www.lncrna2target.org) (19) is a web-based tool that stores lncRNA-gene pairs which were obtained from the independent low throughput, such as qRT-PCR and western blot analysis, or high throughput, such as gene microarray and RNA-Seq. In the present study, various IncRNAs which may regulate the DEGs were obtained from the LncRNA2Target database and the IncRNA-gene regulatory network was constructed and visualized by Cytoscape software (20).

\section{Results}

Quality control and mapping. As a representative, the quality control (QC) outcome of the RNA-Seq reads of the Hep3B cells, which was obtained from FastQC (http://www.bioinformatics.babraham.ac.uk/projects/fastqc/) is shown in Fig. 1. An average of 95.5\% (93.0-96.6\%) mapping rate of each RNA-Seq sample was obtained from TopHat and the mapping rate for each sample is shown in Table I.

Differential expression analysis. Based on Cufflinks, the mapped reads in each sample were assembled into transcripts, and the number of reads mapped to the genes was calculated. Fig. 2 shows the number of reads mapped to every gene between Hep3B and Hep3B-C, as well as Huh7 and Huh7-C. Thus, it should be unbiased to conduct differential expression analysis based on RPKM in the four samples. By Cuffdiff, a number of 218 DEGs containing 82 downregulated and 136 upregulated DEGs were identified in the Hep3B-C vs. Hep3B samples. In addition, a total of 591 DEGs containing 298 downregulated and 293 upregulated DEGs were identified in the Huh7-C vs. Huh7 samples. A total of 22 overlaps were found between these two lists of DEGs (Table II). Among these, 16 overlaps were consistently upregulated or downregulated in the Hep3B-C and Huh7-C samples.

Enriched functions and pathways. Through functional and pathway enrichment analysis, several GO terms were found to be co-enriched in the two lists of DEGs, such as 'steroid metabolic process' and 'cholesterol metabolic process'. The top 20 most significantly enriched GO terms in the two lists of DEGs are shown in Fig. 3. In addition, two pathways, which include 'arginine and proline metabolism' and 'glycine, serine and threonine metabolism', were found to be enriched in the DEGs of Hep3B-C. The 7 enriched pathways of DEGs of Huh7-C are shown in Table III. 


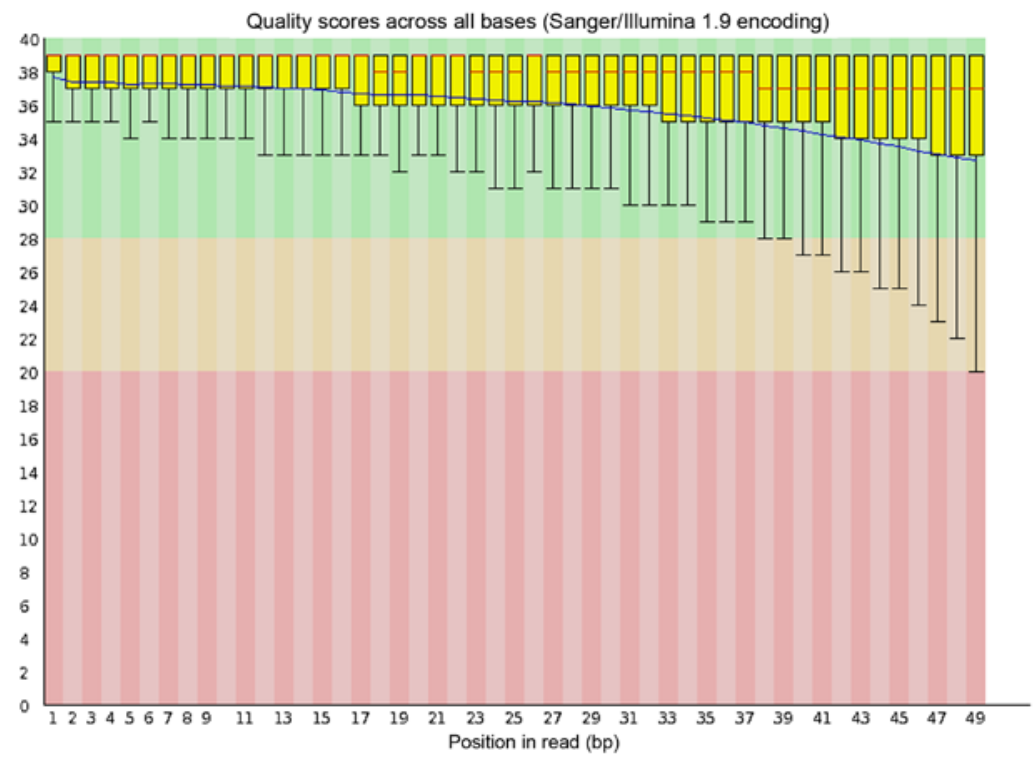

Figure 1. Quality scores across all bases in the Hep3B samples. The blue, yellow and red areas indicate high, moderate and low quality of bases in the corresponding positions, respectively.
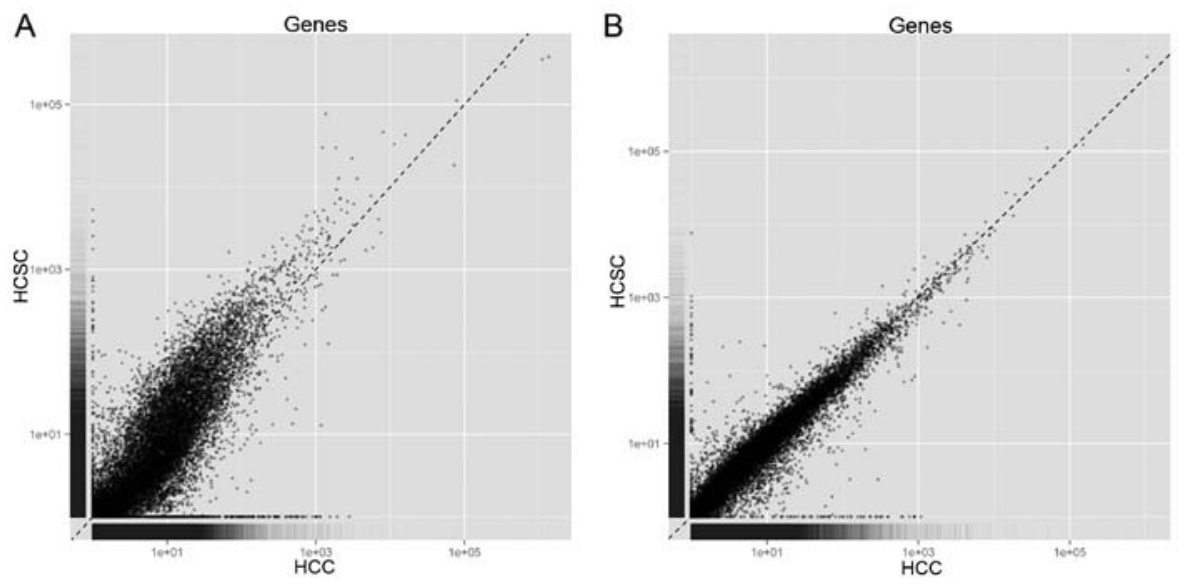

Figure 2. Number of reads mapped to every gene in the (A) Hep3B and Hep3B-C and (B) Huh7 and Huh7-C samples.

Table II. Overlapped genes identified between the DEGs in the Hep3B-C and Huh7-C samples.

\begin{tabular}{|c|c|c|c|c|c|}
\hline Symbol & Log2FC_Hep3B-C & Log2FC_Huh7-C & Symbol & Log2FC_Hep3B-C & Log2FC_Huh7-C \\
\hline FXYD3 $^{a}$ & -5.98 & -3.09 & SPRY4 ${ }^{b}$ & 3.53 & 1.28 \\
\hline CACNB2 & -4.71 & 2.53 & $\mathrm{BMP} 4^{\mathrm{b}}$ & 3.63 & 1.35 \\
\hline HDAC9 & -4.15 & 7.61 & $\mathrm{BOP} 1^{\mathrm{b}}$ & 3.67 & 1.27 \\
\hline IFI6 $6^{\mathrm{a}}$ & -3.93 & -3.03 & HSD3B7 & 3.70 & -1.36 \\
\hline TNS $1^{\mathrm{a}}$ & -3.83 & -2.31 & $\mathrm{NQO}^{\mathrm{b}}$ & 3.87 & 1.96 \\
\hline PRTG & -3.61 & 1.89 & $\mathrm{MVD}^{\mathrm{b}}$ & 4.06 & 1.32 \\
\hline NTS & -3.28 & 1.45 & UGT1A $1^{\mathrm{b}}$ & 4.37 & 7.02 \\
\hline $\mathrm{KNG}^{\mathrm{a}}$ & -3.15 & -2.05 & SLC43A $2^{\mathrm{b}}$ & 4.50 & 2.02 \\
\hline MAT1A & 3.27 & -2.71 & $\mathrm{HPD}^{\mathrm{b}}$ & 4.56 & 2.20 \\
\hline LY6E & 3.32 & 1.24 & $\mathrm{FASN}^{\mathrm{b}}$ & 4.61 & 1.51 \\
\hline FDFT $1^{\text {b }}$ & 3.39 & 1.12 & VSNL1 $1^{\mathrm{b}}$ & 4.88 & 3.95 \\
\hline
\end{tabular}

$\mathrm{P}<0.05$ was considered to indicate a significant difference. DEGs that were consistent ${ }^{\mathrm{a}}$ downregulated and ${ }^{\mathrm{b}}$ upregulated in the Hep3B-C and Huh7-C samples, respectively. DEGs, differentially expressed genes; Log2FC_Hep3B-C, $\log 2\left(\mathrm{RPKM}_{\mathrm{HCSC}} / \mathrm{RPKM}_{\mathrm{HCC}}\right)$ of Hep3B-C samples; Log2_FC_Huh7-C, $\log 2\left(\mathrm{RPKM}_{\mathrm{HCSC}} / \mathrm{RPKM}_{\mathrm{HCC}}\right)$ of Huh7-C samples. 

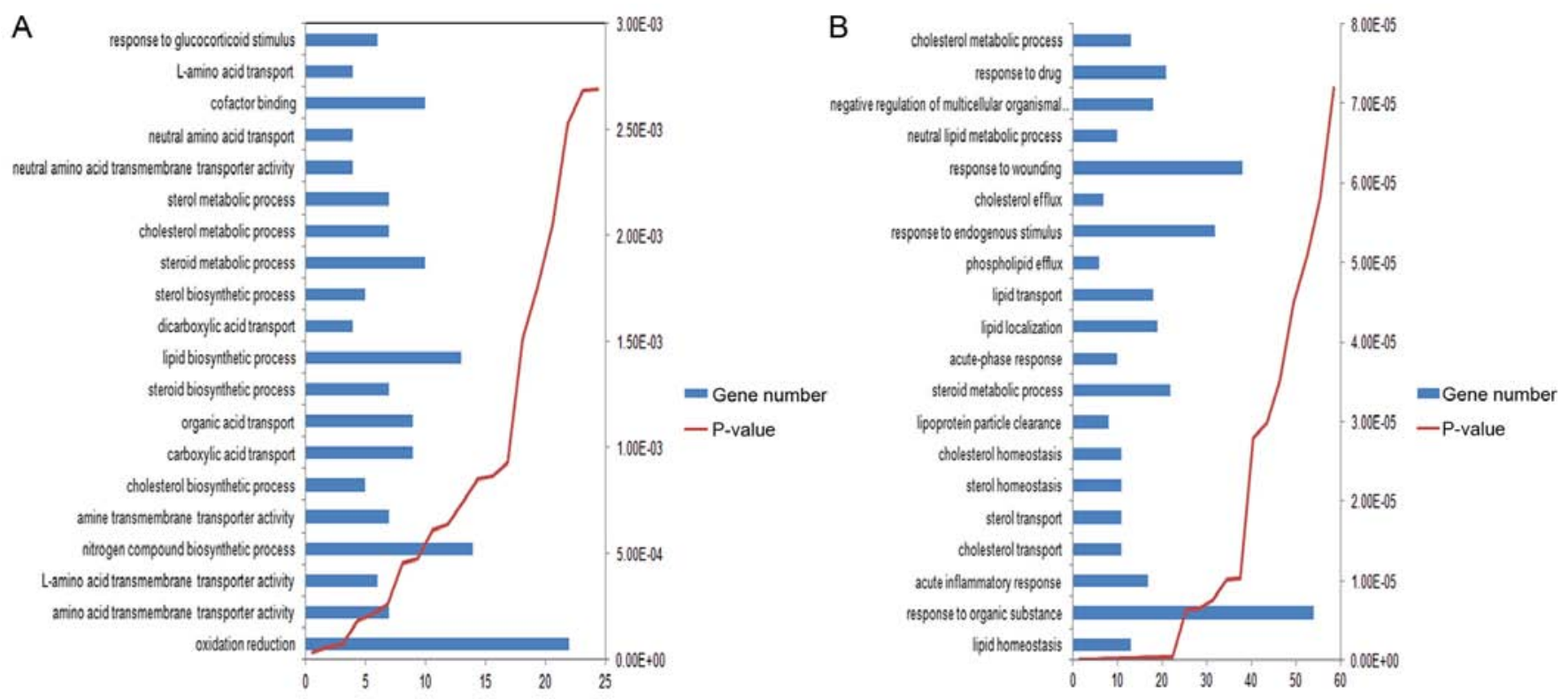

Figure 3. The top 20 most significantly enriched GO terms of the DEGs in the (A) Hep3B-C and (B) Huh7-C samples. GO, Gene Ontology; DEGs, differentially expressed genes.

Table III. Pathways enriched in the DEGs of the Huh7-C samples.

\begin{tabular}{|c|c|c|c|}
\hline Pathway name & No. of genes & P-value & Genes \\
\hline Complement and coagulation cascades & 10 & 0.00111 & $\begin{array}{l}\text { KNG1, C8B, F12, A2M, C3, SERPINF2, CD46, } \\
\text { F2, SERPING1, PROC }\end{array}$ \\
\hline Axon guidance & 13 & 0.00352 & $\begin{array}{l}\text { ROCK2, GNAI1, EFNA1, ABLIM3, EPHB3, } \\
\text { CXCL12, EPHB2, EPHB6, NFAT5, SEMA3D, } \\
\text { SEMA3C, ROBO3, SEMA3A }\end{array}$ \\
\hline PPAR signaling pathway & 8 & 0.0154 & $\begin{array}{l}\text { APOA2, CD36, APOA1, HMGCS2, APOC3, } \\
\text { ACSL4, CPT1A, PCK1 }\end{array}$ \\
\hline TGF- $\beta$ signaling pathway & 9 & 0.0172 & $\begin{array}{l}\text { INHBB, BMP4, EP300, ID2, ROCK2, INHBE, } \\
\text { ID1, ZFYVE9, FST }\end{array}$ \\
\hline Terpenoid backbone biosynthesis & 4 & 0.0177 & HMGCS2, MVD, HMGCR, HMGCS1 \\
\hline Leukocyte transendothelial migration & 10 & 0.0354 & $\begin{array}{l}\text { ITGAL, VAV3, CLDN4, ROCK2, GNAI1, } \\
\text { PECAM1, CLDN2, ESAM, CXCL12, CLDN14 }\end{array}$ \\
\hline Synthesis and degradation of ketone bodies & 3 & 0.0437 & HMGCS2, OXCT1, HMGCS1 \\
\hline
\end{tabular}

DEGs, differentially expressed genes; no. of genes, the number of DEGs contained in each pathway.

lncRNA-gene pairs. In the LncRNA2Target database, $10 \mathrm{lncRNAs}$ were found to regulate 13 of the 16 DEGs with consistent differential expression in the Hep3B-C and Huh7-C samples. Among them, 42 lncRNA-gene pairs were identified. By Cytoscape, the IncRNA-gene regulatory network (Fig. 4) was constructed. lincFOXF1 was the hub node in the network and regulated 7 genes. It may act as a target which influences the transformation of HCC cells to HCSCs.

\section{Discussion}

The process of transformation of HCC cells to hepatocellular carcinoma stem cells (HCSCs) is associated with the initiation, progression, metastasis and poor prognosis of $\operatorname{HCC}(21,22)$.
Yet, the specific mechanism remains unclear. In the present study, through reanalysis of RNA-Seq dataset in GEO, we identified various key factors that may contribute to the development of HCSCs and serve as new therapeutic targets in the treatment of HCC.

Hep3B and Huh7 are two types of HCC cells which have been used in many studies $(23,24)$. In the dataset of this study, HCSCs (Hep3B-C, Huh7-C) were cultured from Hep3B and Huh7 cells. Then, the total RNA of these four samples was extracted and sequenced by Illumina Genome Analyzer II. Here, we identified various DEGs in the Hep3B-C vs. Hep3B and Huh7-C vs. Huh7 samples, respectively. A total of 22 overlaps were obtained between these two lists of DEGs. In addition, 16 genes were found to display consistent differential 


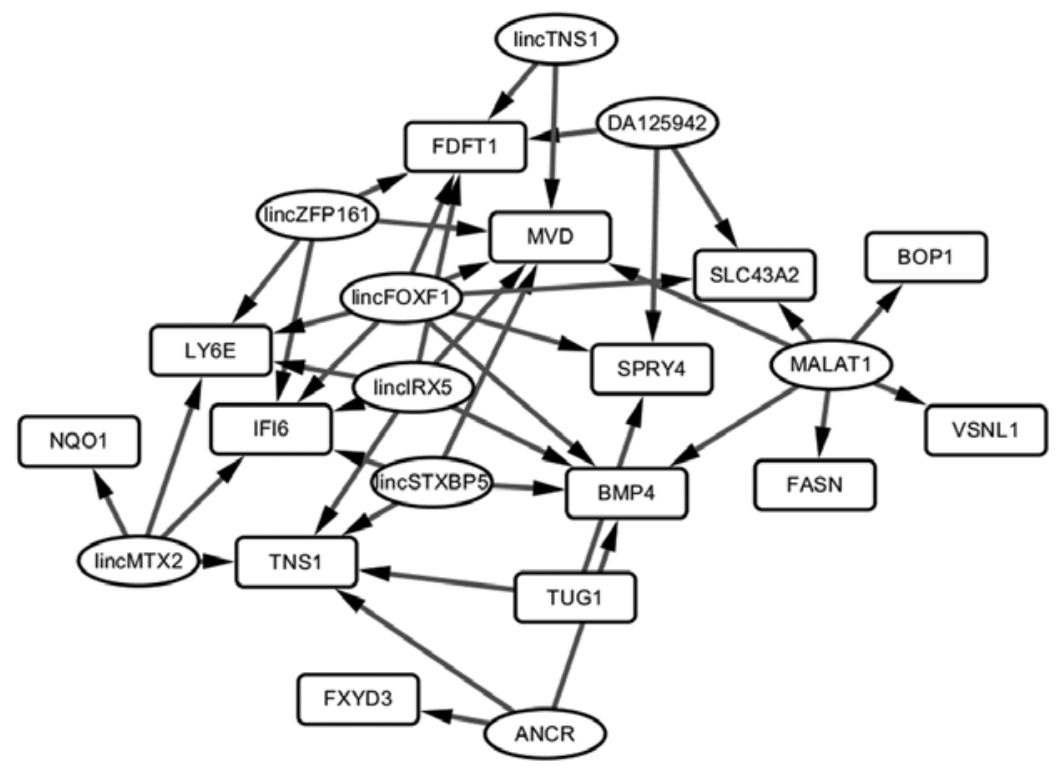

Figure 4. The lncRNA-gene regulation network of genes that was found to have consistent differential expression in the Hep3B-C and Huh7-C samples. The ovals and squares indicate lncRNAs and genes, respectively. lncRNA, long non-coding RNA.

expression in the Hep3B-C and Huh7-C samples. These results indicate that various common mechanisms exist in the development of HCSC samples from different HCC cells which is consistent with previous studies $(25,26)$. FXYD3 was one of the most downregulated genes in both HCSC samples among the 16 identified genes. FXYD3, also known as MTA8 and PLML, encodes a cell membrane protein which regulates the function of ion pumps and ion channels. It also plays an important role in tumor progression (27-29). The upregulation of FXYD3 is associated with many types of cancers, such as rectal cancer, breast cancer, endometrial cancer and intrahepatic cholangiocarcinoma (30-33). In the present study, FXYD3 was found to be downregulated in the Hep3B-C and Huh7-C samples, which has not been demonstrated by any other previous study. This indicates its role in the transformation of HCSCs from HCC cells and the association with the development of HCC.

The pathways of 'substance metabolism and transport', including 'steroid metabolic process' and 'cholesterol metabolic process' were found to be enriched in the two lists of DEGs. As steroid is an important metabolite, the dysregulation of its functions is associated with the generation of CSCs in many types of cancers and could affect progression (34-36). In this study, $M V D$, which is related to cholesterol biosynthesis, was found to be present in both of these two pathways: 'steroid biosynthetic/metabolic process' and 'terpenoid backbone biosynthesis' in the Huh7-C sample. To date, there is no study that shows the relationship between $M V D$ and the development of HCC. Thus, this gene may be a potential novel biomarker.

IncRNAs are a type of non-coding RNAs which populate in the cancer genome. They play an important role in gene translation and have been identified as potential biomarkers in many types of cancers (37). Moreover, the rapid growth of high-throughput technologies, such as microarray and RNA-Seq, has accelerated the mining of novel lncRNAs followed by the vast emergence of new resources. For example,
NONCODE 2016 (http://www.bioinfo.org/noncode/), which was developed by Zhao et al, provides the largest amount of lncRNA collections and annotation information, including conservation annotation and references (38). As a manually curated lncRNA database with experimental support, Lnc2Cancer (http://www.bio-bigdata.net/lnc2cancer) could provide the relationship between 1 ncRNAs and various human cancers (39). In the present study, LncRNA2Target curated lncRNA-gene pairs through manipulating low- and high-throughput experiments to screen the potential lncRNAs for the 16 DEGs which displayed consistent differential expression in the two types of HCSCs. In the IncRNA-gene regulatory network, we found that lincFOXF1 regulated 7 DEGs and was identified as a hub node. lincFOXF1 is also known as FENDRR and its downregulation was found to be associated with gastric cancer cell metastasis and poor prognosis (40). It also plays an important role in mammalian embryogenesis (41), and has been closely associated with stem cell development. Thus, it may also affect the transformation of CSCs and provide valuable targets for cancer treatment. Moreover, the targets of lincFOXF1, including BMP4, FDFT1, IFI6, LY6E, MVD, SLC43A2 and SPRY4, have been validated as playing roles in cancer progression and metastasis, and several were novel targets identified in the present study. Yet, further studies are still needed.

In conclusion, through the analysis of the RNA-Seq dataset in GEO, we identified various potential targets that may contribute to the generation of HCSCs and are associated with the initiation, progression and metastasis of HCC. These targets may facilitate the diagnosis and treatment of $\mathrm{HCC}$ and improve the prognosis of $\mathrm{HCC}$ patients.

\section{Acknowledgements}

We would like to thank all the members of our research group for their enthusiastic participation in this study. 


\section{References}

1. Chen X, Liu HP, Li M and Qiao L: Advances in non-surgical management of primary liver cancer. World J Gastroenterol 20: 16630-16638, 2014.

2. Margini C and Dufour JF: The story of HCC in NAFLD: From epidemiology, across pathogenesis, to prevention and treatment. Liver Int 36: 317-324, 2016.

3. Chen JG and Zhang SW: Liver cancer epidemic in China: Past, present and future. Semin Cancer Biol 21: 59-69, 2011.

4. D'Alessandro R, Messa C, Refolo MG and Carr BI: Modulation of sensitivity and resistance to multikinase inhibitors by microenvironmental platelet factors in HCC. Expert Opin Pharmacother 16: 2773-2780, 2015.

5. Zou H, Feng X and Cao JG: Twist in hepatocellular carcinoma: Pathophysiology and therapeutics. Hepatol Int 9: 399-405, 2015.

6. Frau M, Biasi F, Feo F and Pascale RM: Prognostic markers and putative therapeutic targets for hepatocellular carcinoma. Mol Aspects Med 31: 179-193, 2010.

7. Todoroki K, Ogasawara S, Akiba J, Nakayama M, Naito Y, Seki N, Kusukawa J and Yano H: CD44v3 ${ }^{+} / \mathrm{CD} 24^{-}$cells possess cancer stem cell-like properties in human oral squamous cell carcinoma. Int J Oncol 48: 99-109, 2016.

8. Ding M, Li J, Yu Y, Liu H, Yan Z, Wang J and Qian Q: Integrated analysis of miRNA, gene, and pathway regulatory networks in hepatic cancer stem cells. J Transl Med 13: 259, 2015.

9. Moitra K: Overcoming multidrug resistance in cancer stem cells. BioMed Res Int 2015: 635745, 2015.

10. Khorrami S, Zavaran Hosseini A, Mowla SJ and Malekzadeh R: Verification of ALDH activity as a biomarker in colon cancer stem cells-derived HT-29 cell line. Iran J Cancer Prev 8: e3446, 2015.

11. Sun J, Luo Q, Liu L, Zhang B, Shi Y, Ju Y and Song G: Biomechanical profile of cancer stem-like cells derived from MHCC97H cell lines. J Biomech 49: 45-52, 2016.

12. Matthai SM and Ramakrishna B: Cancer stem cells in hepatocellular carcinoma - an immunohistochemical study with histopathological association. Indian J Med Res 142: 391-398, 2015.

13. Jones MF, Hara T, Francis P, Li XL, Bilke S, Zhu Y, Pineda M, Subramanian M, Bodmer WF and Lal A: The CDX1microRNA-215 axis regulates colorectal cancer stem cel differentiation. Proc Natl Acad Sci USA 112: E1550-E1558, 2015

14. Wang M, Ren D, Guo W, Huang S, Wang Z, Li Q, Du H, Song L and Peng X: N-cadherin promotes epithelial-mesenchymal transition and cancer stem cell-like traits via ErbB signaling in prostate cancer cells. Int J Oncol 48: 595-606, 2016.

15. Gui X, Li H, Li T, Pu H and Lu D: Long noncoding RNA CUDR regulates HULC and beta-catenin to govern human liver stem cell malignant differentiation. Mol Ther 23: 1843-1853, 2015.

16. Li H, An J, Wu M, Zheng Q, Gui X, Li T, Pu H and Lu D: LncRNA HOTAIR promotes human liver cancer stem cell malignant growth through downregulation of SETD2. Oncotarget 6: 27847-27864, 2015

17. Trapnell C, Roberts A, Goff L, Pertea G, Kim D, Kelley DR, Pimentel H, Salzberg SL, Rinn JL and Pachter L: Differential gene and transcript expression analysis of RNA-seq experiments with TopHat and Cufflinks. Nat Protoc 7: 562-578, 2012.

18. Huang W, Sherman BT and Lempicki RA: Systematic and integrative analysis of large gene lists using DAVID bioinformatics resources. Nat Protoc 4: 44-57, 2009.

19. Jiang Q, Wang J, Wu X, Ma R, Zhang T, Jin S, Han Z, Tan R, Peng J, Liu G, et al: LncRNA2Target: A database for differentially expressed genes after lncRNA knockdown or overexpression. Nucleic Acids Res 43: D193-D196, 2015.

20. Shannon P, Markiel A, Ozier O, Baliga NS, Wang JT, Ramage D, Amin N, Schwikowski B and Ideker T: Cytoscape: A software environment for integrated models of biomolecular interaction networks. Genome Res 13: 2498-2504, 2003.

21. Li J, Yu Y, Wang J, Yan Z, Liu H, Wang Y, Ding M, Cui L, Wu M, Jiang X, et al: Establishment of a novel system for the culture and expansion of hepatic stem-like cancer cells. Cancer Lett 360: $177-186,2015$

22. You N, Zheng L, Liu W, Zhong X, Wang W and Li J: Proliferation inhibition and differentiation induction of hepatic cancer stem cells by knockdown of BC047440: A potential therapeutic target of stem cell treatment for hepatocellular carcinoma. Oncol Rep 31: 1911-1920, 2014.
23. Yao CJ, Yeh CT, Lee LM, Chuang SE, Yeh CF, Chao WJ, Lai TY and Lai GM: Elimination of cancer stem-like 'side population' cells in hepatoma cell lines by chinese herbal mixture 'tien-hsien liquid'. Evid Based Complement Alternat Med 2012: 617085 , 2012.

24. Chen YL, Yuan RH, Yang WC, Hsu HC and Jeng YM: The stem cell E3-ligase Lin-41 promotes liver cancer progression through inhibition of microRNA-mediated gene silencing. J Pathol 229: 486-496, 2013

25. Yang Z, Zhang L, Ma A, Liu L, Li J, Gu J and Liu Y: Transient mTOR inhibition facilitates continuous growth of liver tumors by modulating the maintenance of $\mathrm{CD}_{133^{+}}$cell populations. PLoS One 6: e28405, 2011.

26. Nishina S, Shiraha H, Nakanishi Y, Tanaka S, Matsubara M, Takaoka N, Uemura M, Horiguchi S, Kataoka J, Iwamuro M, et al: Restored expression of the tumor suppressor gene RUNX3 reduces cancer stem cells in hepatocellular carcinoma by suppressing Jagged1-Notch signaling. Oncol Rep 26: 523-531, 2011.

27. Zhu ZL, Zhao ZR, Zhang Y, Yang YH, Wang ZM, Cui DS, Wang MW, Kleeff J, Kayed H, Yan BY, et al: Expression and significance of FXYD-3 protein in gastric adenocarcinoma. Dis Markers 28: 63-69, 2010.

28. Wang MW, Gu P, Zhang ZY, Zhu ZL, Geng Y, Kayed H, Zentgraf $\mathrm{H}$ and Sun XF: FXYD3 expression in gliomas and its clinicopathological significance. Oncol Res 18: 133-139, 2009.

29. Widegren E, Onnesjö S, Arbman G, Kayed H, Zentgraf H, Kleeff J, Zhang H and Sun XF: Expression of FXYD3 protein in relation to biological and clinicopathological variables in colorectal cancers. Chemotherapy 55: 407-413, 2009.

30. Herrmann P and Aronica SM: Estrogen and tamoxifen up-regulate FXYD3 on breast cancer cells: Assessing the differential roles of ER $\alpha$ and ZEB1. Springerplus 4: 245, 2015.

31. Li Y, Zhang X, Xu S, Ge J, Liu J, Li L, Fang G, Meng Y, Zhang H and Sun X: Expression and clinical significance of FXYD3 in endometrial cancer. Oncol Lett 8: 517-522, 2014.

32. Loftås P, Onnesjö S, Widegren E, Adell G, Kayed H, Kleeff J, Zentgraf $\mathrm{H}$ and Sun XF: Expression of FXYD-3 is an independent prognostic factor in rectal cancer patients with preoperative radiotherapy. Int J Radiat Oncol Biol Phys 75: 137-142, 2009.

33. Subrungruanga I, Thawornkunob C, ChawalitchewinkoonPetmitrc P, Pairojkul C, Wongkham S and Petmitrb S: Gene expression profiling of intrahepatic cholangiocarcinoma. Asian Pac J Cancer Prev 14: 557-563, 2013.

34. Hilton $\mathrm{HN}$ and Clarke CL: Impact of progesterone on stem/progenitor cells in the human breast. J Mammary Gland Biol Neoplasia 20: 27-37, 2015.

35. Finlay-Schultz J and Sartorius CA: Steroid hormones, steroid receptors, and breast cancer stem cells. J Mammary Gland Biol Neoplasia 20: 39-50, 2015.

36. Callari M, Guffanti A, Soldà G, Merlino G, Fina E, Brini E, Moles A, Cappelletti V and Daidone MG: In-depth characterization of breast cancer tumor-promoting cell transcriptome by RNA sequencing and microarrays. Oncotarget 7: 976-994, 2016.

37. Sahu A, Singhal U and Chinnaiyan AM: Long noncoding RNAs in cancer: From function to translation. Trends Cancer 1: 93-109, 2015.

38. Zhao Y, Li H, Fang S, Kang Y, Wu W, Hao Y, Li Z, Bu D, Sun N, Zhang MQ, et al: NONCODE 2016: An informative and valuable data source of long non-coding RNAs. Nucleic Acids Res 44: D203-D208, 2016.

39. Ning S, Zhang J, Wang P, Zhi H, Wang J, Liu Y, Gao Y, Guo M, Yue M, Wang L, et al: Lnc2Cancer: A manually curated database of experimentally supported lncRNAs associated with various human cancers. Nucleic Acids Res 44: D980-D985, 2016.

40. Xu TP, Huang MD, Xia R, Liu XX, Sun M, Yin L, Chen WM, Han L, Zhang EB, Kong R, et al: Decreased expression of the long non-coding RNA FENDRR is associated with poor prognosis in gastric cancer and FENDRR regulates gastric cancer cell metastasis by affecting fibronectin 1 expression. J Hematol Oncol 7: 63, 2014.

41. Grote P and Herrmann BG: The long non-coding RNA Fendrr links epigenetic control mechanisms to gene regulatory networks in mammalian embryogenesis. RNA Biol 10: 1579-1585, 2013. 